\title{
Elevated B Cell Activation is Associated with Type 2 Diabetes Development in Obese Subjects
}

\author{
Xiao Zhai ${ }^{\mathrm{a}}$ Guangfang Qian ${ }^{\mathrm{b}}$ Yiran Wang ${ }^{\mathrm{a}}$ Xiao Chen ${ }^{\mathrm{a}}$ Jiancan Luc \\ Yaping Zhang ${ }^{c}$ Qin Huang ${ }^{c}$ Qijin Wang ${ }^{c}$ \\ aGraduate Management Unit, Changhai hospital, Second Military Medical University, Shanghai, \\ 'Department of Endocrinology, Zhangqiu Municipal Hospital of Traditional Chinese Medicine, \\ Zhangqiu, 'Department of Endocrinology, Changhai hospital, Second Military Medical University, \\ Shanghai, China
}

\section{Key Words}

B cell • Type 2 diabetes • Obesity

\begin{abstract}
Background/Aims: Despite strong association between obesity and the pathogenesis of type 2 diabetes (T2D), only a subset of obese individuals eventually develops T2D. We sought to determine the immunological factors behind this heterogeneity. Methods: Peripheral blood of obese non-diabetic subjects and obese diabetic subjects were collected and the B cell responses in these subjects were analyzed. Results: We found that the $B$ cells from obese diabetic subjects had similar B cell subtype composition and secreted similar levels of low-grade pro-inflammatory cytokines to obese non-diabetic subjects, characteristic to the background chronic immune activation frequently observed in obese subjects. When examining adaptive B cell antibody responses, however, obese diabetic subjects presented much higher levels of polyclonal activation and antibody secretion, with impaired ability to response to new antigens such as seasonal influenza vaccination. Conclusions: These data demonstrated that in obese diabetic subjects, B cell adaptive response is impaired and potentially contribute to overall higher inflammation.

\section{Introduction}

Type 2 diabetes (T2D) is an increasingly prevalent chronic metabolic disorder characterized by hyperglycemia in the context of insulin resistance, affecting close to 300 million people worldwide, and is associated with a series of complications [1,2]. The current paradigm explains that a series of conditions caused by overnutrition and obesity, including increased oxidative stress, amyloid deposition in pancreas and ectopic lipid deposition in muscle, pancreas and liver, is the leading cause for insulin resistance and inlet beta-cell
\end{abstract} X. Zhai, G. Qian and Y. Wang contributed equally to the work. 


\section{Cellular Physiology Cell Physiol Biochem 2016;38:1257-1266

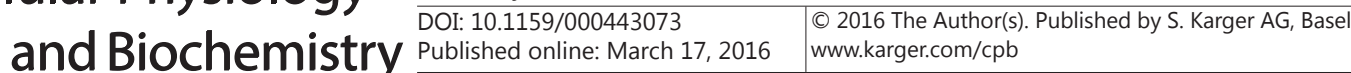 \\ Zhai et al.: B Cell Activation and Diabetes}

dysfunction in T2D [2]. However, despite overwhelmingly strong association of obesity and diabetes in people of European or African descents, in the East Asian population, obesity and diabetes are only moderately associated, with only about $30 \%$ of diabetic cases being obese and only about one-third of obese insulin-resistant individuals eventually develop T2D [3]. Genetic and epigenetic factors, as well as immunological correlates, such as previous infection history, diet and intestinal microbe composition, were thought to explain this heterogeneity $[2,4]$.

Recently, the role of immune system in T2D has been increasingly studied [5]. A host of T2D risk factors, such as smoking, high fat and high carbohydrate diet, high blood pressure, and obesity, were found to establish a more pro-inflammatory immune environment in the individual. Higher concentrations of pro-inflammatory cytokines, including TNF- $\alpha$, IL-6, IL$1 \beta$, CCL2, as well as increased kinase activation, were found in the adipose tissues of obese individuals [6-8]. In addition, studies have shown that B cells in T2D were found to regulate inflammation through producing pro-inflammatory cytokines such as IL- 6 and TNF- $\alpha$ and promote pro-inflammatory $\mathrm{T}$ cell functions $[9,10]$. Whether differences in B cell responses contribute to diabetes pathogenesis in obesity is unclear.

In this study, we attempted to elucidate the role of B cells in pathogenesis of diabetes in obese individuals. We compared and contrasted the B cell function in lean healthy subjects (healthy), obese subjects with no sign of diabetes (obese), and obese subjects diagnosed with T2D (obese diabetic). We found that in both obese groups, the B cells exhibited a more proinflammatory phenotype, which included increase of activated plasmablasts and antibodies of IgM and IgG subtypes, than those in lean subjects. Marked differences in antibody responses were found between obese subjects and obese diabetic subjects. Obese diabetic subjects contained higher frequencies of antibody-secreting cells than healthy subjects, but the number of exogenous antigen-specific antibody-secreting cell was not increased, which is often observed in polyclonal B cell activation. Furthermore, impaired de novo B cell response toward novel antigen stimulation were observed in obese diabetic but not obese subjects. Together, these data demonstrated that B cell response from obese diabetic subjects were defective compared to obese subjects, a feature not previously observed.

\section{Materials and Methods}

\section{Study subjects}

A total of 15 obese diabetic subjects and 15 obese non-diabetic subjects were recruited at Changhai Hospital. Patients were classified under our strict study criteria. Only subjects with fasting glucose concentration $\geq 7.0 \mathrm{mmoL} / \mathrm{L}$ were considered as diabetic. All obese subjects suffer from class I obesity (30 $\mathrm{kg} / \mathrm{m}^{2}<\mathrm{BMI}<35 \mathrm{~kg} / \mathrm{m}^{2}$ ). Individuals with other complications that might affect their immune system, such as those with chronic history of smoking, concurrent other infections, and presence of other chronic diseases, were excluded from the study. Fifteen lean healthy individuals were recruited from staff in the hospital (18 $\mathrm{kg} / \mathrm{m}^{2}<\mathrm{BMI}<23 \mathrm{~kg} / \mathrm{m}^{2}$ ). All study subjects are between the ages of 45 to 58 . No significant differences in age and sex between study groups were found. Some of the obese and obese diabetic subjects received 2012 2013 unadjuvated seasonal influenza vaccine containing hemagglutinin antigens from A/California/7/2009 (H1N1)pdm09-like virus, A/Victoria/361/2011 (H3N2)-like virus and B/Wisconsin/1/2010-like virus and followed up for 8 weeks. All participants signed informed consent forms upon enrolment and experiments were performed in accordance with the Declaration of Helsinki. This study was approved by Changhai Hospital ethics committee.

Sample preparation and cell culture

Peripheral blood (50 - $100 \mathrm{~mL}$ ) was collected into heparinized tubes by venous puncture. PBMCs were isolated from leukopheresis samples with standard Ficoll-Hypaque procedure and frozen immediately at $-80^{\circ} \mathrm{C}$ for $1 \mathrm{~d}$ and transferred to $-150^{\circ} \mathrm{C}$ until use. Culture medium was made with RPMI 1640 supplemented with $10 \%$ fetal cow serum, $100 \mathrm{U}$ penicillin and $0.1 \mathrm{mg} / \mathrm{mL}$ streptomycin, and $2 \mathrm{mM}$ L-glutamine. In some experiments, stimulating agents, including anti-human CD40 antibody (eBioscience), anti-human CD19 


\section{Cellular Physiology Cell Physiol Biochem 2016;38:1257-1266 and Biochemistry Published online: March 17, $2016 \quad$\begin{tabular}{l|l} 
DOI: 10.1159/000443073 & $\begin{array}{l}\text { (c) } 2016 \text { The Author(s). Published by S. Karger AG, Basel } \\
\text { www.karger.com/cpb }\end{array}$
\end{tabular} \\ Zhai et al.: B Cell Activation and Diabetes}

(BCR) (BioLegend, San Diego, CA), E. coli LPS 055:B5 (Sigma, St. Louis, M0), and/or CpG-B (sequence TCG TCG TTT TGT CGT TTT GTC GTT) at were added accordingly.

Flow cytometry

Monoclonal antibodies specific to CD3, CD19, CD20, CD21, CD27, CD40 (ebioscience, San Diego, CA) were used with concentrations specified by the manufacturer. Cells were thawed and cultured overnight in medium, then stained with surface antibodies and Violet Dead Cell Stain (Invitrogen) according to procedures provided by the manufacturer. Results were analyzed using FlowJo software (Tree Star, Ashland, OR)

\section{Cell isolation}

B cells were isolated using Human B cell Negative Selection Kit (Stemcell, Vancouver, Canada) according to manufacturer's protocol.

\section{ELISA assay}

Purified B cells were cultured in medium or with $0.5 \mu \mathrm{g} / \mathrm{mL}$ anti-human CD40 and $0.5 \mu \mathrm{g} / \mathrm{mL}$ antihuman CD19 antibodies for $72 \mathrm{~h}$, after which culture supernatant were collected. The concentrations of IL-6 (standard curve range: 2 - 200 pg/mL), IL-10 (standard curve range: 2 - 300 pg/mL) and TNF- $\alpha$ (standard curve range: $4-500 \mathrm{pg} / \mathrm{mL}$ ) were determined in culture supernatants using commercial ELISA kits (eBioscience, San Diego, CA), according to the manufacturer's protocols. The concentrations of tetanus toxin-specific IgM, IgG and IgA antibodies in the serum were measured by Serion ELISA classic tetanus kits (Virion \Serion, Würzburg, Germany).

\section{ELISPOT assay}

PBMCs were cultured in medium with $1 \mu \mathrm{g} / \mathrm{mL}$ LPS and $2.5 \mu \mathrm{g} / \mathrm{mL} \mathrm{CpG}$ at $37^{\circ} \mathrm{C} 5 \% \mathrm{CO}_{2}$ for 3 d. For the preparation of ELISPOT plates, 96-well PVDF plates (Pall Life Sciences, Port Washington, NY) were first primed with $50 \mu \mathrm{L} / \mathrm{mL}$ ethanol, quickly washed with PBS, then coated with $5 \mu \mathrm{g} / \mathrm{mL}$ of goat anti-human kappa (Rockland, Limerick, PA) and goat anti-human lambda (Rockland), $5 \mu \mathrm{g} / \mathrm{mL}$ deactivated tetanus toxin, or $5 \mu \mathrm{g} / \mathrm{mL}$ of each hemagglutinin from 2012-2013 seasonal flu vaccine overnight. Plates were then washed with PBS and blocked with culture medium for $2 \mathrm{~h}$ to eliminate nonspecific binding. Cells were then plated at 200,000 cells per well for $12 \mathrm{~h}$, after which the plates were washed. Bound antibodies were detected with alkaline phosphate (AP)-conjugated anti-human IgM, AP-conjugated anti-human IgG, or horseshoe radish peroxidase (HRP)-conjugated anti-human IgA (KPL, Gaithersburg, MD). Spots were developed using 3-amino-9-ethylcarbazole substrate kit or Vector Blue substrate kit (Vector Labs, Burlington, Canada) according to manufacturer's protocols.

\section{Statistical analysis}

Kruskal-Wallis one-way analysis of variance (ANOVA) was used for comparisons between multiple groups and then Dunn's test was used for pair-wise comparisons. Student's t test was used for comparison between two groups. Wilcoxon matched-pairs test was used for comparison of paired data from the same individual. All statistical analyses were done using Prism (GraphPad Software, San Diego, CA). P < 0.05 was considered significant.

\section{Results}

$B$ cells from obese subjects and obese diabetic subjects exhibited more activated phenotype than healthy subjects

When encountering a specific antigen, naïve B cell and resting memory B cells can be activated and differentiate into antibody-producing plasmablasts, which are larger in size, more granular, and present $\mathrm{CD} 19^{\mathrm{lo}} \mathrm{CD} 27^{\mathrm{hi}}$ surface expression [11]. Thus, the presence of $\mathrm{CD} 19^{\mathrm{lo}} \mathrm{CD} 27^{\text {hi }}$ plasmablasts in circulation is frequently used as a marker for B cell activation during an inflammation [12]. We first sought to determine the B cell activation levels of obese and obese diabetic subjects, and how they compare to lean healthy subjects. Peripheral blood mononuclear cells (PBMCs) were collected from study groups and B cell surface marker 


\section{Cellular Physiology Cell Physiol Biochem 2016;38:1257-1266 \\ \begin{tabular}{l|l} 
and Biochemistry Published online: March 17, 2016 & $\begin{array}{l}\text { D } 2016 \text { The Author(s). Published by S. Karger AG, Basel } \\
\text { www.karger.com/cpb }\end{array}$
\end{tabular} \\ Zhai et al.: B Cell Activation and Diabetes}

expression were analyzed with flow cytometry. As shown in Fig. 1A, B cells from obese and obese diabetic subjects tended to be higher in side scatter and more granular than those from healthy subjects. Obese subjects and obese diabetic subjects also contained higher percentage of B cells in the lymphocyte compartment (Fig. 1B). When examining B cell surface marker expression, shown in Fig. 1C and 1D, healthy subjects contained mostly CD19+CD27- naïve $\mathrm{B}$ cells, $\mathrm{CD} 19^{+} \mathrm{CD} 27^{+}$memory B cells, with very little $\mathrm{CD} 19^{\mathrm{lo}} \mathrm{CD} 27^{\text {hi }}$ plasmablasts, while both obese subjects and obese diabetic subjects had lower percentages of $C D 19^{+} \mathrm{CD} 27^{-}$naïve $\mathrm{B}$ cells and $\mathrm{CD} 19^{+} \mathrm{CD} 27^{+}$memory B cells and higher percentages of $\mathrm{CD} 19^{\mathrm{lo}} \mathrm{CD} 27^{\text {hi }}$ plasmablasts. The absolute numbers of $\mathrm{CD} 19^{+} \mathrm{CD} 27^{-}$naïve $\mathrm{B}$ cells and $\mathrm{CD} 19^{+} \mathrm{CD} 27^{+}$memory B cells were not significantly different between the three groups, but the absolute numbers of $\operatorname{CD} 19^{\text {lo }} \mathrm{CD} 27^{\mathrm{hi}}$ plasmablasts were significantly higher in obese and obese diabetic subjects (Fig. 1E). No significant differences were seen between obese non-diabetic and obese diabetic groups. Together, these data demonstrated that obesity in general induced higher levels of B cell activation with higher frequencies of plasmablasts.

$B$ cells from obese subjects and obese diabetic subjects showed higher production of proinflammatory cytokines

Previously, B cells in T2D were found to exhibit a more inflammatory cytokine expression profile and promote $\mathrm{T}$ cell inflammation, thus potentially involved in T2D pathogenesis [9]. We next sought to determine whether the B cell cytokine expression is differentially regulated between obese diabetic subjects and obese non-diabetic subjects. Pure B cells were isolated from PBMCs through negative selection, with greater than 95\% purity (Fig. $2 \mathrm{~A}$ ), and then cultured in medium for $72 \mathrm{~h}$. Supernatant was then taken and enzyme-linked immunosorbent assay (ELISA) was performed. IL-6 is a pleiotropic cytokine secreted by B cells, is involved in pro-inflammatory responses and metabolic processes, and is implicated in T2D pathogenesis $[9,13]$. TNF- $\alpha$ is another cytokine that can be produced by effector $B$ cells and has multiple functions in inducing inflammation [14]. As shown in Fig. 2B, both obese and obese diabetic subjects had higher IL-6 secretion from B cells than healthy subjects, either when cultured in medium directly ex vivo, or when cultured with anti-CD40 and anti-B cell receptor (BCR) antibodies to simulate BCR signaling. In terms of TNF- $\alpha$, both obese subjects and obese diabetic subjects had higher TNF- $\alpha$ secretion than healthy subjects when cultured in medium ex vivo, while under CD40/BCR stimulation, B cells from all three groups presented high concentrations of TNF- $\alpha$.

Regulatory B cells are important immune response regulators in suppressing excessive $\mathrm{T}$ cell-mediated inflammation in autoimmune diseases and chronic infections [15]. IL-10 is pivotal in regulatory B cell function. We analyzed the IL-10 production by B cells, and found that B cells from obese subjects secreted more IL-10 than those from healthy subjects ex vivo (Fig. 2C). On the other hand, although obese diabetic subjects have a trend to secrete more IL-10 ex vivo, the result was not statistically significant. When stimulated with anti-CD40 and anti-BCR antibodies, B cells from obese subjects secreted comparable levels of IL-10 with healthy subjects, while the IL-10 secretion from B cells purified from obese diabetic subjects was significantly lowered. This suggests that B cells in obese diabetic subjects may have impaired ability to secrete IL-10, even under CD40/BCR-stimulated conditions.

Together, these data suggest that although B cells from both obese non-diabetic and obese diabetic subjects in general present a more pro-inflammatory cytokine secretion profile than those from lean healthy subjects, with a potential IL-10 production defect in B cells from obese diabetic subjects.

Antibody response in obese and obese diabetic subjects was less antigen-specific

Since both obese groups contained higher frequency of plasmablasts in PBMCs, we decided to measure the antibody secretion from these study groups. B cells were co-cultured with LPS and CpG for $3 \mathrm{~d}$ to stimulate antibody production, after which the total IgM, IgG and IgA production and antigen-specific antibody production were measured by enzymelinked immunospot assay (ELISPOT). Anti-Ig lambda and anti-Ig kappa antibodies were used 


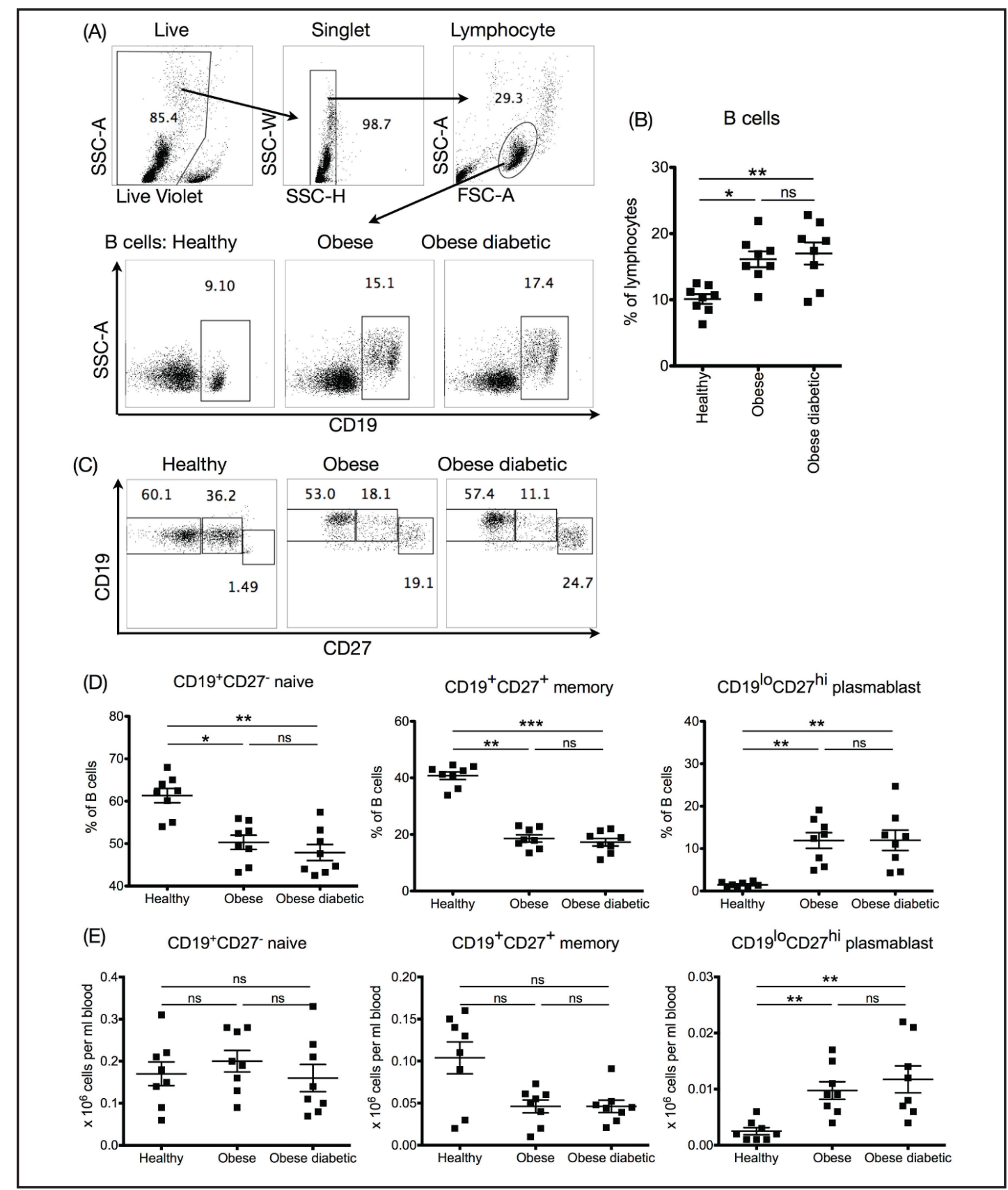

Fig. 1. B cell composition and surface marker expression in healthy, obese, and obese diabetic subjects. (A) Identification of B cells from healthy, obese, and obese diabetic subjects. Frozen PBMCs from study subjects were thawed and stained with surface marker antibodies. The gating of B cells of a representative from each of the three study groups was shown. (B) The percentage of B cells in total lymphocytes in all three study groups. (C) B cell composition of a representative from each of the three study groups. The gatings of CD $19^{+} \mathrm{CD} 27^{-}$naïve B cells (left), CD $19^{+} \mathrm{CD} 27^{+}$memory B cells (middle) and CD $19^{\text {lo }} \mathrm{CD} 27^{\text {hi }}$ plasmablasts (lower right) were shown. (D) The frequencies of B cell subsets in all three groups. (E) The absolute numbers of B cell subsets in all three groups *: $\mathrm{P}<0.05$. **: $\mathrm{P}<0.01$. ***: $\mathrm{P}<0.001$. ns: not significant (Kruskal-Wallis oneway ANOVA and Dunn's test). Line and error bars represent mean \pm s.e.m.

to coat the ELISPOT plate to capture total antibody secreting cells (ASCs), while since all our subjects were previously vaccinated against tetanus toxin, tetanus toxin were used as a model for exogenous antigen. The number of spots on the plate represents the number 
(A)

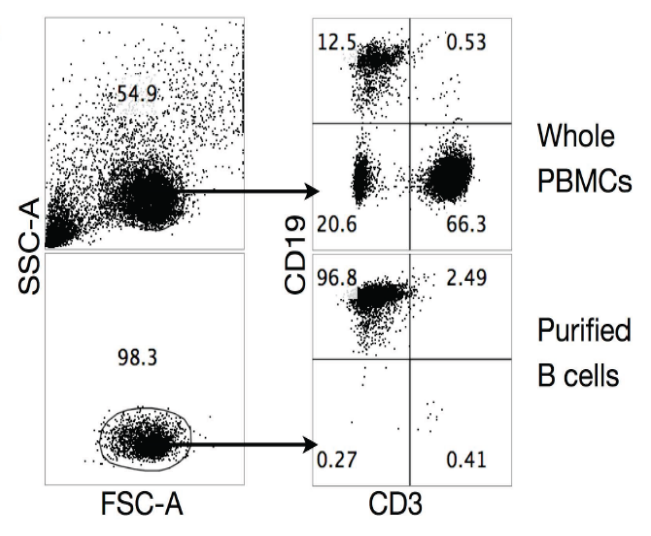

(B)

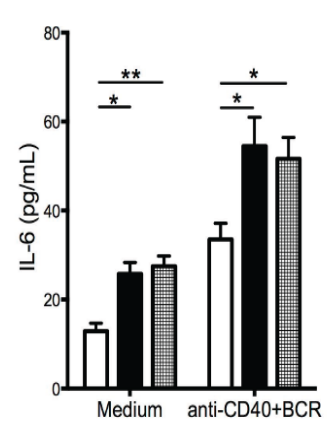

TNF-a

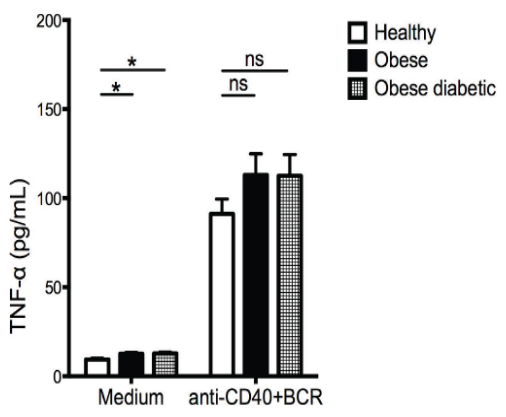

(C)

IL-10

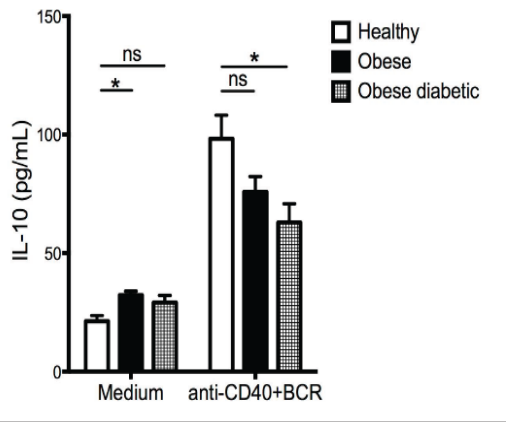

Fig. 2. B cell cytokine secretion from healthy, obese, and obese diabetic subjects. B cells were isolated from thawed PBMCs by negative selection using magnetic beads, then cultured in medium without or with anti-CD40 and anti-BCR antibodies for 72h, after which the supernatant was collected and cytokine concentrations were determined with ELISA. Data were obtained from 8 subjects in each group, in triplicate wells. (A) Efficacy of B cell purification in one representative subject. (B) IL- 6 and TNF- $\alpha$ secretion of all three study groups. (C) IL-10 secretion of all three study groups. *: P < 0.05. **: P < 0.01. ns: not significant (Kruskal-Wallis one-way ANOVA and Dunn's test). Error bar represents s.e.m.

of ASCs. As shown in Fig. 3A, obese diabetic subjects contained more total IgM and total IgG ASCs than those from healthy subjects, while obese subjects tended to contain higher concentrations of total IgM ASCs but the trend was not statistically significant. No significant differences in total IgA production were found between study groups.

Although total IgM and IgG secretion was higher in obese diabetic subjects, this trend was not observed when the tetanus toxin-specific antibodies were measured. As shown in Fig. 3B, no differences between study groups were found in terms of tetanus toxin-specific IgM, IgG, or IgA ASCs. These data suggest that although obese diabetic subjects secreted higher levels of antibodies, tetanus toxin-specific antibody concentrations were not increased proportionally. The specificities of excess ASCs were unknown. Percentage-wise, tetanus toxin-specific antibody-secreting cells were less represented in the total antibodysecreting cells (Fig. 3C). We also examined the tetanus toxin-specific antibody concentration in the serum, and discovered that the obese diabetic group had significantly lower level of tetanus toxin-specific IgG (Fig. 3D). Despite this, all subjects had tetanus toxin-specific IgG concentration $>1.0 \mathrm{IU} / \mathrm{mL}$, the accepted threshold of immune protection [16].

Obese diabetic subjects had reduced ability to produce de novo antibody responses

Due to the lowered percentage of antigen-specific antibody secreting cells in obese diabetic subjects, we decided to examine whether this group had impaired ability to induce 
Fig. 3. Total and antigen-specific antibody secreting cells from healthy, obese and obese diabetic subjects. PBMCs were cultured in medium for $3 \mathrm{~d}$ in the presence of CpG and LPS to stimulate B cells toward antibody-secreting cells. ELISPOT plates were coated with either anti-lambda and anti-kappa antibodies, or with tetanus toxin. The stimulated cells were then placed in the plate for $12 \mathrm{~h}$ before being washed off. The bound antibodies were then detected by HRP- or AP-conjugated anti-human IgM, IgG or IgA and HRP or AP substrates. Number of antibody-secreting cells (ASCs) was counted as the number of spots on the plate. (A) Total IgM-, IgGand IgA-secreting cells in all three study groups. (B)
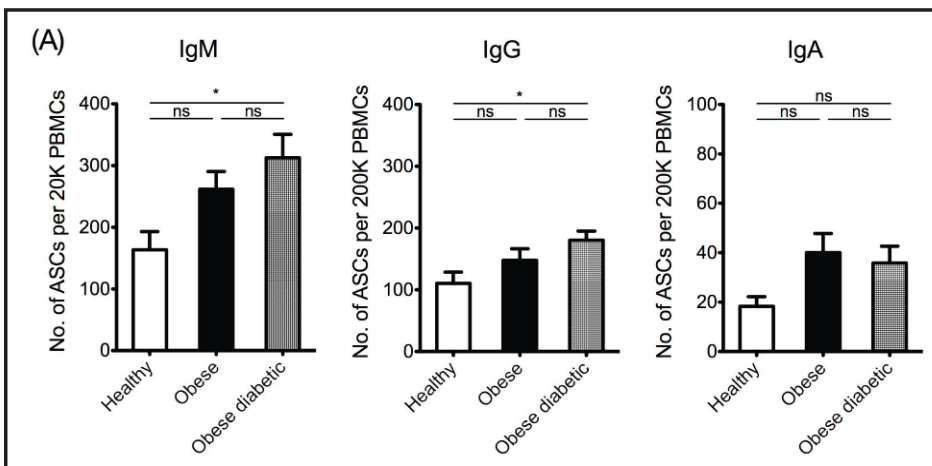

Anti-Ig Lambada

(B)
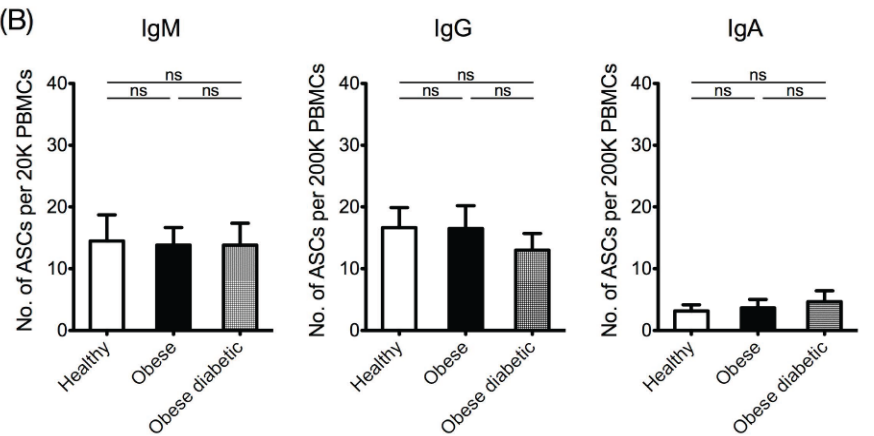

Tetanus Toxin

(C)

Tetanus toxin-specific / total ASC

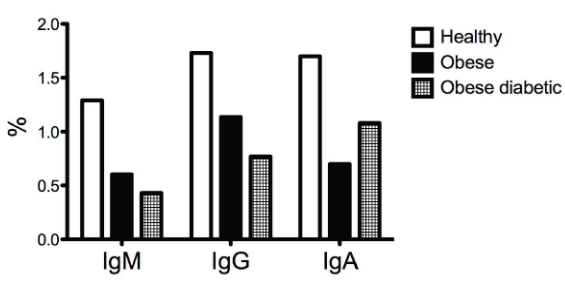

(D)
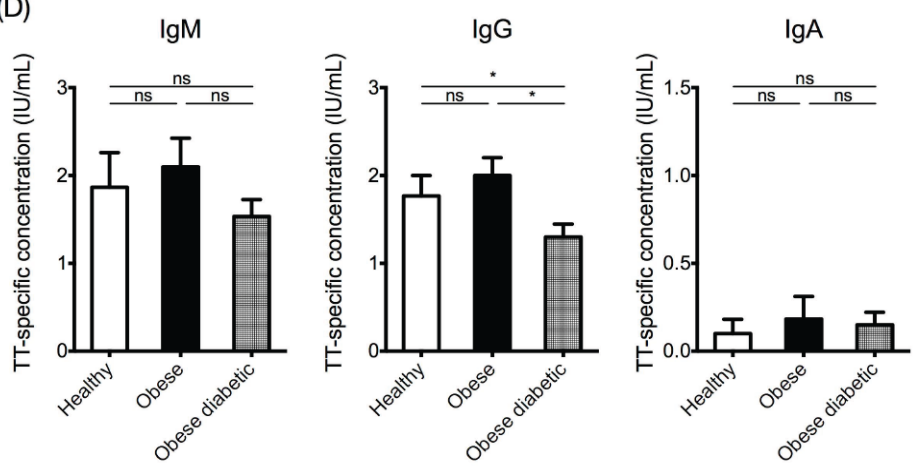

Tetanus toxin-specific IgM-, IgG- and IgA-secreting cells in all three study groups. (C) The percentage of tetanus toxin-specific antibody-secreting cells in all subjects, obtained by the number of tetanus toxin-specific cells divided by the total number. (D) Serum tetanus toxin (TT)-specific antibody concentration in all three study groups. *: P < 0.05. ns: not significant (Kruskal-Wallis one-way ANOVA and Dunn's test). Error bar represents s.e.m.

de novo antibody response toward new exogenous antigens. 6 subjects from each study group received 2012-2013 seasonal influenza vaccine at Week 0 . The background flu-specific antibody response at Week 0 and the de novo flu-specific antibody response at week 8 from these subjects were measured by ELISPOT. Although the total amount of flu-specific antibody 


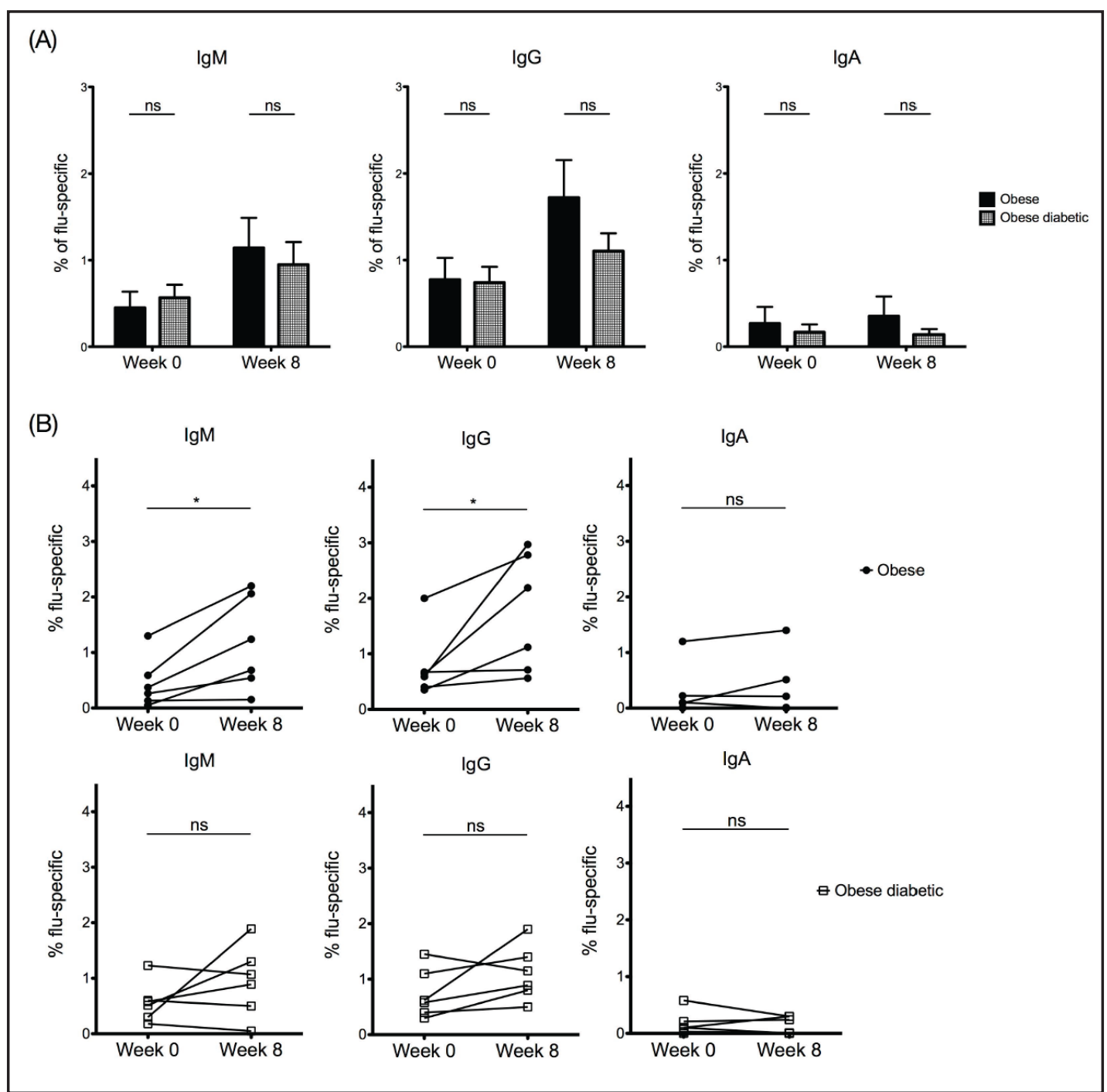

Fig. 4. De novo antibody response to flu vaccination of obese and obese diabetic subjects. Prior to performing ELISPOT, 6 subjects from each group were vaccinated at Week 0. Peripheral blood samples were collected at Week 0 and Week 8. ELISPOT plates were coated with either anti-lambda and anti-kappa antibodies, or with the hemagglutinin antigens contained in the vaccine. (A) The percentage of flu-specific IgM-, IgG- or IgA-secreting cells from each group at Week 0 and Week 8, calculated by the number of flu-specific spots divided by the total number. ns: not significant (Student's t test). (B) The percentage of flu-specific IgM-, IgG- or IgA-secreting cells from each individual at Week 0 and Week 8. Filled circles: obese subjects. Open squares: obese diabetic subjects. *: P < 0.05. ns: not significant. (Wilcoxon matched-pairs test).

production between obese subjects and obese diabetic subjects were comparable in both week 0 and week 8 (Fig. 4A), interesting response patterns were found when we examined the response patient by patient. We paired each subject's own background (Week 0) and Week 8 flu-specific antibody production, and examined whether the subject responded to the vaccination. As shown in Fig. 4B, obese subjects had successfully mounted anti-flu response such that the Week 8 flu-specific IgM and IgG production is significantly higher than background response at Week 0 . In obese diabetic subjects, however, no significant differences between Week 8 and Week 0 were observed. Together, these data suggested that although obese diabetic subjects appeared to have similar levels of B cell inflammation with obese subjects, the B cells from obese diabetic subjects failed to respond to novel antigens. A higher dose may be required for successful vaccination in this group. 


\section{Cellular Physiology Cell Physiol Biochem 2016;38:1257-1266 \\ \begin{tabular}{l|l} 
and Biochemistry Published online: March 17, 2016 & $\begin{array}{l}\text { Do } 2016 \text { The Author(s). Published by S. Karger AG, Basel } \\
\text { www.karger.com/cpb }\end{array}$
\end{tabular} \\ Zhai et al.: B Cell Activation and Diabetes}

\section{Discussion}

In this study, we demonstrated several differences between the B cell responses in obese subjects with no sign of diabetes (obese) and obese subjects with diabetes. First, although B cells from both groups secreted more pro-inflammatory IL- 6 and TNF- $\alpha$, only B cells from obese diabetic subjects had defect in upregulating regulatory cytokine IL-10, a pivotal cytokine in mediating immune suppression by regulatory B cells $[15,17]$. Second, although B cells form both groups were composed of more activated subsets, with lower frequencies of $\mathrm{CD} 19^{+} \mathrm{CD} 27^{-}$naïve $\mathrm{B}$ cells and $\mathrm{CD} 19^{+} \mathrm{CD} 27^{+}$memory $\mathrm{B}$ cells, and higher frequencies of $\mathrm{CD} 19^{\mathrm{lo}} \mathrm{CD} 27^{\mathrm{hi}}$ plasmablasts, B cells from obese diabetic subjects contained higher concentrations of total IgM and IgG without proportional increase of exogenous antigen-specific antibody production. Furthermore, B cells from obese diabetic subjects had impaired response to novel antigen stimulation through flu vaccination, while obese subjects responded to flu vaccination normally. These data showed that although B cells from obese diabetic subjects appeared to have higher activation levels, the antibody response was actually impaired.

$B$ cell-mediated immune response and regulation are important participants in immune response. Antigen-specific antibody production from activated B cells, plasmablasts and plasma cells provide the first line of defense against exogenous pathogens in exposed surfaces, by binding and neutralizing pathogens, facilitate phagocytosis for destruction and antigen-presentation. On the other hand, self-reactive antibody production is involved in destruction of self tissues and initiation of autoimmune diseases $[18,19]$. Thus, regulating B cell responses during inflammation is critically important. Ideally, antigen-specific B cells are activated when encountering a foreign antigen, then receive helper signals from follicular helper T cells (Tfh) and undergo further proliferation and differentiation to become either specialized antibody-secreting plasmablasts or memory B cells that specialize in rapid response in second encounter [20]. In several chronic inflammatory diseases, however, polyclonal B cell activation was observed through a mechanism called bystander effect (i.e., the excess pro-inflammatory signals activate irrelevant B cell clones), and feeds back to excessive inflammation through effector B cell functions [21, 22]. How polyclonal activation of B cells and its subsequent regulatory roles impact the pathogenesis of T2D is currently unknown. Presumably, the higher activation level of B cells of unknown specificity may in turn induce higher inflammation within the patient in a positive feedback loop.

Previously, Frasca et al. showed an enhancement of anti-vaccine IgG concentration in elderly diabetic individuals compared to elderly healthy individuals [23], whereas our study showed no significant increase in the frequencies of flu-specific B cells in obese diabetic individuals aftervaccination. A couple of differences might have accounted for this discrepancy. First, the study by Frasca et al. used the 2011-2012 vaccine strains, which were the same as the 2010-2011 vaccine strains and potentially had the problem of crossreactivity by B cells from the year before. We used the 2012-2013 vaccine strains, which were different from the 2011-2012 strains. And second, the demography of our study subjects was different, with no Asian diabetic individuals, while all our subjects were Chinese. This might also accounted for the discrepancies between this and a previous study by Jagannathan et al. [10], in which the authors have found a reduction of TNF- $\alpha$ and IL-10 secretion by unstimulated B cells from diabetic patients.

Together, data presented here showed that albeit higher activation level, in obese subjects with no diabetes, B cell response could still function normally, while in obese diabetic subjects, several B cell defects, including lower IL-10 production and ineffective antibody response, were observed. Currently, it is unclear if this phenomenon resulted from diabetes development, or contributed to diabetes pathogenesis in obesity. Since interaction with Tfh is critical to mediating the selection of antigen-specific B cells though survival, proliferation and differentiation, future studies may focus on the role of Th cells in obesity and further elucidate the precise mechanism in T2D development. 


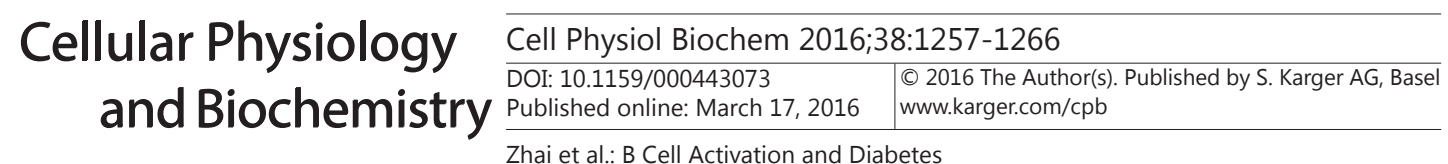

\section{Acknowledgments}

This study was supported by the 1255 Scientific Project of Changhai Hospital (NO.: CH125541900).

\section{Disclosure Statement}

None.

\section{References}

1 Smyth S, Heron A: Diabetes and obesity: the twin epidemics. Nat Med 2006;12:75-80.

2 Leahy JL: Pathogenesis of type 2 diabetes mellitus. Arch Med Res 2005;36:197-209.

3 Donath MY, Shoelson SE: Type 2 diabetes as an inflammatory disease. Nat Rev Immunol 2011;11:98-107.

4 Manna P, Jain SK: Phosphatidylinositol-3,4,5-triphosphate and cellular signaling: implications for obesity and diabetes. Cell Physiol Biochem 2015;35:1253-75.

5 Donath MY: Targeting inflammation in the treatment of type 2 diabetes: time to start. Nat Rev Drug Discov 2014;13:465-476.

6 Busque SM, Stange G, Wagner CA: Dysregulation of the glutamine transporter Slc38a3 (SNAT3) and ammoniagenic enzymes in obese, glucose-intolerant mice. Cell Physiol Biochem 2014;34:575-89.

7 Gregor MF, Hotamisligil GS: Inflammatory mechanisms in obesity. Annu Rev Immunol 2011;29:415-445.

8 Shoelson SE, Herrero L, Naaz A: Obesity, inflammation, and insulin resistance. Gastroenterology 2007;132:2169-2180.

9 DeFuria J, Belkina AC, Jagannathan-Bogdan M, Snyder-Cappione J, Carr JD, Nersesova YR, Markham D, Strissel KJ, Watkins AA, Zhu M, Allen J, Bouchard J, Toraldo G, Jasuja R, Obin MS, McDonnell ME, Apovian C, Denis GV, Nikolajczyk BS: B cells promote inflammation in obesity and type 2 diabetes through regulation of T-cell function and an inflammatory cytokine profile. Proc Natl Acad Sci USA 2013;110:5133-5138.

10 Jagannathan M, McDonnell M, Liang Y, Hasturk H, Hetzel J, Rubin D, Kantarci A, Van Dyke TE, Ganley-Leal LM, Nikolajczyk BS: Toll-like receptors regulate B cell cytokine production in patients with diabetes. Diabetologia 2010;53:1461-1471.

11 Bordon Y: B cell responses: Plasmablasts walk the line. Nat Rev Immunol 2010;10:541.

12 Fink K: Origin and Function of Circulating Plasmablasts during Acute Viral Infections. Front Immunol 2012;3:78.

13 Scheller J, Chalaris A, Schmidt-Arras D, Rose-John S: The pro- and anti-inflammatory properties of the cytokine interleukin-6. Biochim Biophys Acta 2011;1813:878-888.

14 Lund FE: Cytokine-producing B lymphocytes-key regulators of immunity. Curr Opin Immunol 2008;20:332-338.

15 Mauri C, Bosma A: Immune regulatory function of B cells. Annu Rev Immunol 2012;30:221-241.

16 Reder S, Riffelmann M, Becker C, von Konig CHW: Measuring immunoglobulin G antibodies to tetanus toxin, diphtheria toxin, and pertussis toxin with single-antigen enzyme-linked immunosorbent assays and a bead-based multiplex assay. Clin Vaccine Immunol 2008;15:744-749.

17 Iwata Y, Matsushita T, Horikawa M, Dilillo DJ, Yanaba K, Venturi GM, Szabolcs PM, Bernstein SH, Magro CM, Williams AD, Hall RP, St Clair EW, Tedder TF: Characterization of a rare IL-10-competent B-cell subset in humans that parallels mouse regulatory B10 cells. Blood 2011;117:530-541.

18 Pillai S, Mattoo H, Cariappa A: B cells and autoimmunity. Curr Opin Immunol 2011;23:721-731.

19 Tan EM: Autoantibodies, autoimmune disease, and the birth of immune diagnostics. J Clin Invest 2012;122:3835-3836.

20 Crotty S: T follicular helper cell differentiation, function, and roles in disease. Immunity 2014;41:529-542.

21 Montes CL, Acosta-Rodriguez EV, Merino MC, Bermejo DA, Gruppi A: Polyclonal B cell activation in infections: infectious agents' devilry or defense mechanism of the host? J Leukoc Biol 2007;82:1027-1032.

22 Moir S, Fauci AS: Insights into B cells and HIV-specific B-cell responses in HIV-infected individuals. Immunol Rev 2013;254:207-224.

23 Frasca D, Diaz A, Romero M, Mendez NV, Landin AM, Ryan JG, Blomberg BB.: Young and elderly patients with type 2 diabetes have optimal B cell responses to the seasonal influenza vaccine. Vaccine 2013;31:3603-3610. 